\title{
Fabrication of a 3 dimensional dielectrophoresis electrode by a metal inkjet printing method
}

\author{
Seung Hyun Lee', Gyu-Young Yun², Yul Koh', Sang-Ho Lee ${ }^{2^{*}}$ and Yong-Kweon Kim*
}

\begin{abstract}
We proposed a micro electrode fabrication method by a metal inkjet printing technology for the bio-applications of dielectrophoresis (DEP). The electrodes are composed of bottom planar gold (Au) electrodes and three dimensional (3D) silver (Ag) electrodes fabricated locally on the Au electrode through metal inkjet printing. We observed the negative DEP characteristics of the $4 \mu \mathrm{m}$ polystyrene beads on the both electrodes at the $500 \mathrm{kHz}, A C 20 \mathrm{~V}_{\mathrm{pp}}$ point. The number of beads trapped on the printed Ag electrode is 79 and 25 on the planar Au electrode because of spatially larger electric field in a 3D electrode system.
\end{abstract}

Keywords: Metal inkjet printing technology; Dielectrophoresis (DEP); 3D electrode

\section{Introduction}

Three dimensional (3D) electrodes are effectively used in a wide range of applications such as electronic circuit components, micro sensors, micro actuators, and microfluidic system. In particular, the 3D electrode is useful for the dielectrophoresis (DEP) of biological samples including cells, proteins, and particles. Compared to the planar electrode, the 3D electrode structure allows it to transmit a large DEP force to the biological samples due to the high gradient electrical field generated between the 3D electrodes [1]. Therefore, the high gradient electrical field in 3D DEP chips could enhance separation and trapping performance. There are many investigations into the trapping and separation of a biological sample using 3D DEP chips.

Conventional microfabrication technologies have several drawbacks in 3D electrode fabrication. It is difficult to form 3D electrodes that have a micrometer scale thickness using thermal evaporation and sputtering. Metal electroplating can be used to fabricate 3D electrodes with high aspect ratios, but complicated procedures are required. The inkjet printing technology has been studied for the patterning of the 3D electrode. Inkjet printing can be directly utilized in fabricating 3D

\footnotetext{
* Correspondence: sholee7@kitech.re.kr; yongkkim@snu.ac.kr

${ }^{2}$ Korea Institute of Industrial Technology, Ansan, Korea

'School of Electrical Engineering and Computer Science, Seoul National University, Seoul, Korea

Full list of author information is available at the end of the article
}

structures with a simple process at short run-time and low cost. The inkjet printing method does not require complicated additional fabrication process steps such as photoresist mold and metal seed layer formation for electroplating, in order to pattern a 3D metal structure. For example, various metals can be directly patterned using inkjet printing on the substrate by consuming a small metal source. Therefore, inkjet printing is a very suitable technology to fabricate a 3D metal structure. However, the inkjet printing technology has not yet been applied to the microfluidic systems due to its low fabrication resolution [2-4].

We propose a metal inkjet printing method for the fabrication of the 3D DEP electrode for micro particles manipulation in a microfluidic chamber. For a high resolution inkjet printing, fluorocarbon (FC) film patterning was utilized to make the ink-phobic boundaries which prevent droplets from spreading and enable selective patterning. A microfluidic chamber was assembled with the electrode chip after printing silver (Ag) 3D electrodes for protecting particles and adjusting for fluid control. The DEP performance was verified using the $4 \mu \mathrm{m}$ polystyrene beads and AC drive voltage control [5-7].

\section{Findings \\ Fabrication process}

The FC film as an ink-spreading barrier was selectively patterned on the gold $(\mathrm{Au})$ film to print thick 3D DEP electrodes in a precisely defined printing area. The fabrication processes are presented in Figure 1. First, the $\mathrm{Au}$ 


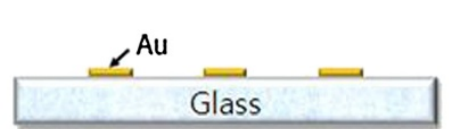

(a)

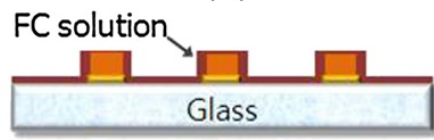

(c)

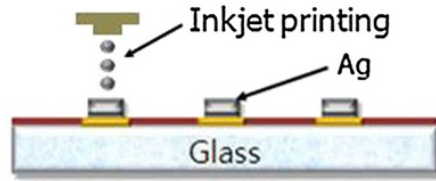

(e)

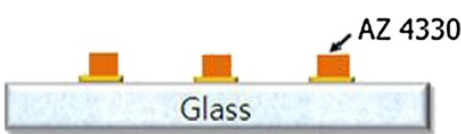

(b)

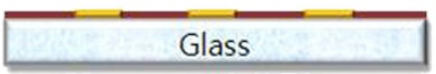

(d)

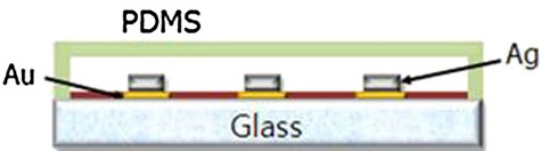

(f)

Figure 1 The process of DEP chip fabrication. (a) Au planar electrode fabrication with lift-off process and Cr/Au thermal evaporation. (b) AZ 4330 PR patterning on the Au electrode surface where the Ag will be printed. (c) Fluorocarbon (FC) coating on the surface and sintering. (d) Removing AZ 4330 PR on the Au electrode. (e) Printing Ag inkjet on the Au electrode surface where FC is not coated. (f) Bonding with PDMS chamber.

planar electrode $(0.3 \mu \mathrm{m}$ thickness $)$ was fabricated on the substrate by a conventional lift-off process by photolithography and $\mathrm{Cr} / \mathrm{Au}$ thermal evaporation (Figure 1(a)). To pattern the surface with hydrophilic and hydrophobic regions selectively, the photoresist (AZ4330) mold was first patterned on the Au bottom electrodes. Then, the fluorocarbon (FC) solution was coated on the surface and photoresist mold (Figure 1(b) and (c)). We used a FC-722 solution diluted with FC-40 (3 M fluorinert ${ }^{\mathrm{Tm}}$ ) at a ratio of 4:1 for surface coating to make the surface hydrophobic. The FC film on the photoresist mold was removed by a lift-off process after curing at $100^{\circ} \mathrm{C}$ for 15 min (Figure 1(d)). Silver was inkjet printed to form thick 3D electrodes inside the printing area as defined by the patterned hydrophobic FC film. Furthermore, the printed $\mathrm{Ag}$ patterns were sintered at $200^{\circ} \mathrm{C}$ for $30 \mathrm{~min}$ to increase the conductivity and strength of adhesion between the ink and substrate (Figure 1(e)). The used printing system is DMP-2800 from Fujifilm Dimatix Corporation and the Ag material is 40LT-15C silver jet ink produced by Advanced Nano Product Corporation. Finally, the PDMS chamber was fabricated by a PDMS molding technology. The chamber and the glass substrate were bonded to each other for the DEP experiments (Figure 1(f)).

Figure 2 shows the printing process to fabricate electrodes on the substrate. In the inkjet printing process (Figure 1(e)), fourteen Ag droplets (about $58.5 \mathrm{pl}$ ) were printed inside a rectangular printing region of $101.6 \mu \mathrm{m} \times$ $3.9 \mathrm{~mm}$ in single printing. The inkjet printing fabrication results are affected by the jetting position and the number of metal inkjet droplets, so it is important to set the droplet jetting condition before the printing process. We simply control the height of an electrode by regulating the overlap-rate of the inkjet jetting.

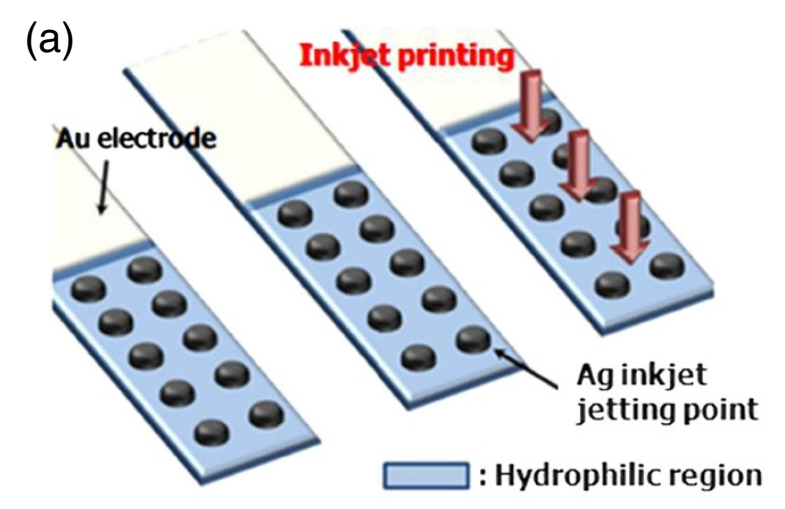

(b)
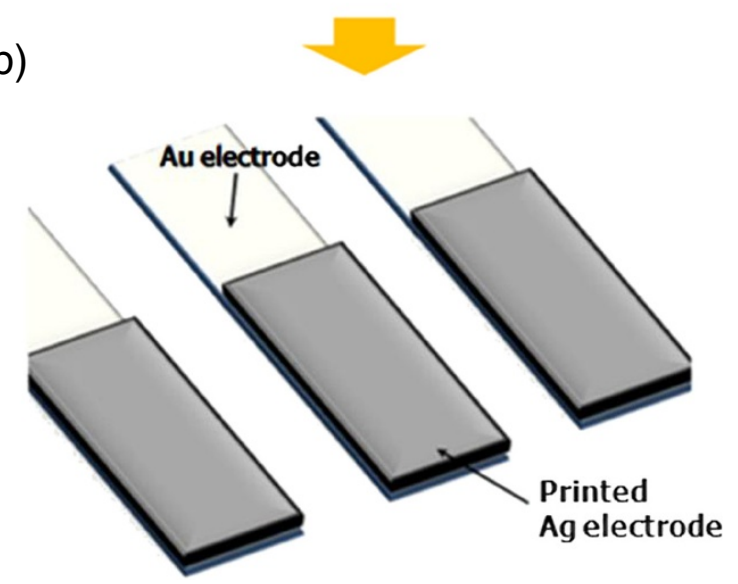

Figure 2 The process of $\mathbf{A g}$ inkjet printing. (a) Several $\mathrm{Ag}$ droplets were jetted inside a rectangular printing region, (b) These small droplets agglomerate together to form larger clusters. 


\section{Inkjet-printed 3D DEP chip design}

Figure 3 shows a schematic of the fabricated 3D DEP chip. The Au planar electrodes are fabricated on the glass wafer. The $\mathrm{Au}$ electrodes are patterned into the shape of a comb drive and used as bottom electrodes. An AC voltage signal is applied to the DEP chip trough the Au electrode. The size of the contact pad connected with the function generator is $2 \mathrm{~mm} \times 2 \mathrm{~mm}$. The gold $(\mathrm{Au})$ rectangular electrodes of $101.6 \mu \mathrm{m} \times 3.9 \mathrm{~mm}$ were interdigitated with a gap of $50.8 \mu \mathrm{m}$. Ag 3D electrodes of $10 \mu \mathrm{m}$ thicknesses are formed on the rectangular $\mathrm{Au}$ electrode on the desired region as shown in Figure 3(b), and the size of the Ag 3D electrode is
101.6 $\mu \mathrm{m} \times 393.7 \mu \mathrm{m}$. The whole dimension of the DEP chip is $3 \mathrm{~cm} \times 1 \mathrm{~cm}$, and the electrodes don't have integer multiple dimensions in meters. This is because the nozzle size is fixed and the jetting inkjet is also restricted to inches. The polydimethylsiloxane (PDMS) chamber is used as a cover to minimize the effects of external contamination as it bonds to the DEP chip. The PDMS chamber has inlet and outlet holes to enable sample injection.

\section{DEP characteristics of the beads}

According to the direction of the DEP force, there are two types for the DEP characteristics: positive and

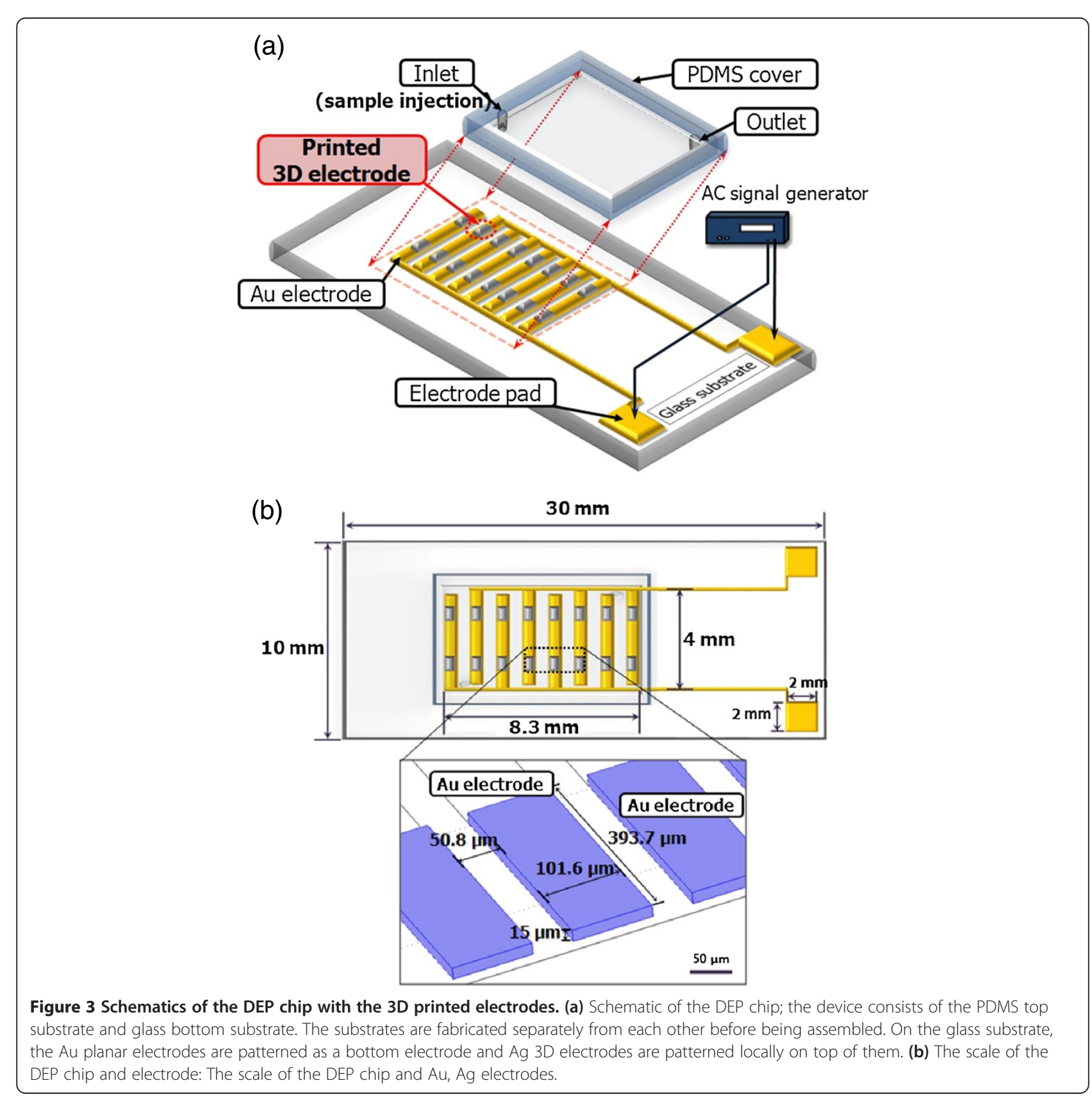


negative DEP. The DEP force is expressed by equation

(1) and (2).

$$
\begin{gathered}
F_{D E P}=2 \pi r^{3} \in_{m} \operatorname{Re}\left[f_{c m}(\omega)\right] \nabla|E|^{2} \quad \omega: \text { angular velocity of the electric field } \\
f_{C M}(\omega)=\left(\frac{\varepsilon_{p}^{\prime}(\omega)-\varepsilon_{m}^{\prime}(\omega)}{\varepsilon_{p}^{\prime}(\omega)+2 \varepsilon_{m}^{\prime}(\omega)}\right) \quad \begin{array}{l}
\varepsilon_{p}: \text { complex permittivity of the particle } \\
\varepsilon_{p}: \text { complex permittivity of the medium }
\end{array}
\end{gathered}
$$

The DEP force is positive when the Clausius-Mossotti $(\mathrm{CM})$ factor $\left(f_{\mathrm{CM}}\right)$ has a positive value while the negative DEP force is generated when $f_{\mathrm{CM}}$ has a negative value. Moreover, the electric field gradient and the radius of the particles are directly affected by the DEP force [8]. According to the electric field distribution, we can predict where the beads are trapped on the chip. In the positive DEP, the particles are gathered toward the higher electric field region while the particles move toward the lower electric field region in the negative DEP. In the case of the polystyrene bead, it has negative DEP characteristics at almost every frequency range.

Figure 4 shows the Finite Element Method (FEM) simulations of the electric field distribution and intensity for the planar electrodes and the 3D electrodes. The electric field distribution is presented in Figure 4(a). The electric field generated between the 3D electrodes has a higher intensity than that between the planar electrodes. As

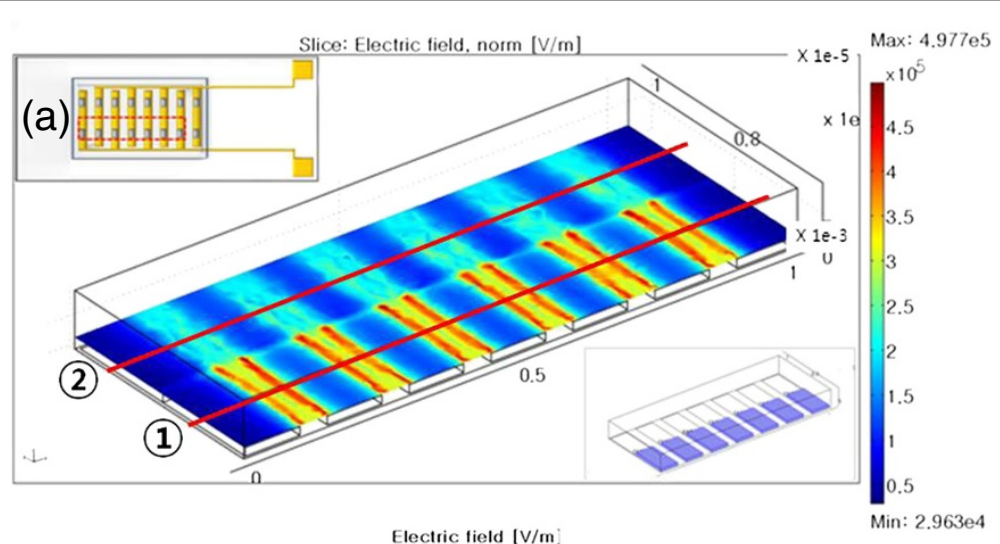

(b)

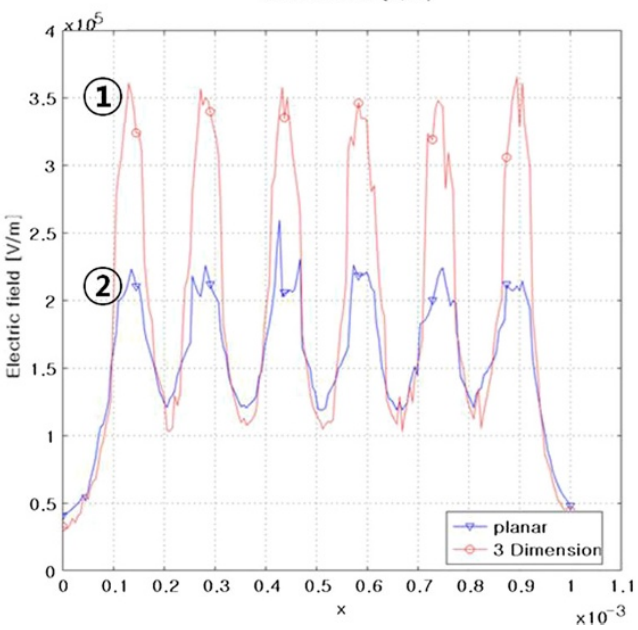

Figure 4 The FEM simulations. (a) The electric field distribution on the electrode plane. (b) The electric field intensity on the electrode plane. Line 1 is on the 3D electrodes and line 2 is on the planar electrodes. The $x$ axis of the simulation range is $0 \mu \mathrm{m}$ to $1000 \mu \mathrm{m}$ and the $y$ axis are $100 \mu \mathrm{m}$ on 3D electrodes and $300 \mu \mathrm{m}$ on planar electrodes. 
shown in Figure 4(b), the electric field gradient for the $3 \mathrm{D}$ electrode is 2 times larger than that for the planar electrode, because the effective electric field range around the $3 \mathrm{D}$ electrode is increased. Therefore, the beads can be affected immensely by the electric field around the $3 \mathrm{D}$ electrode and the trapping rate of the beads is also higher around the 3D electrode than around the planar electrode [9-11].

\section{Results and discussion}

Inkjet printing is inherently problematic in that it is a solution based fabrication process; the ejected ink droplet shows a different spreading behavior under different surface wettability after deposition onto substrates. Different spreads of the ink droplet make it difficult to apply the dimensional control of micron scale patterns of the inkjet printing to MEMS process. We solve this issue by separating non-printing areas and printing areas through patterning FC film on ink-wettable substrates, because the FC film is well known to have low surface energy and poor wettability with various solutions. We can trap jetted Ag ink droplets only inside the ink-
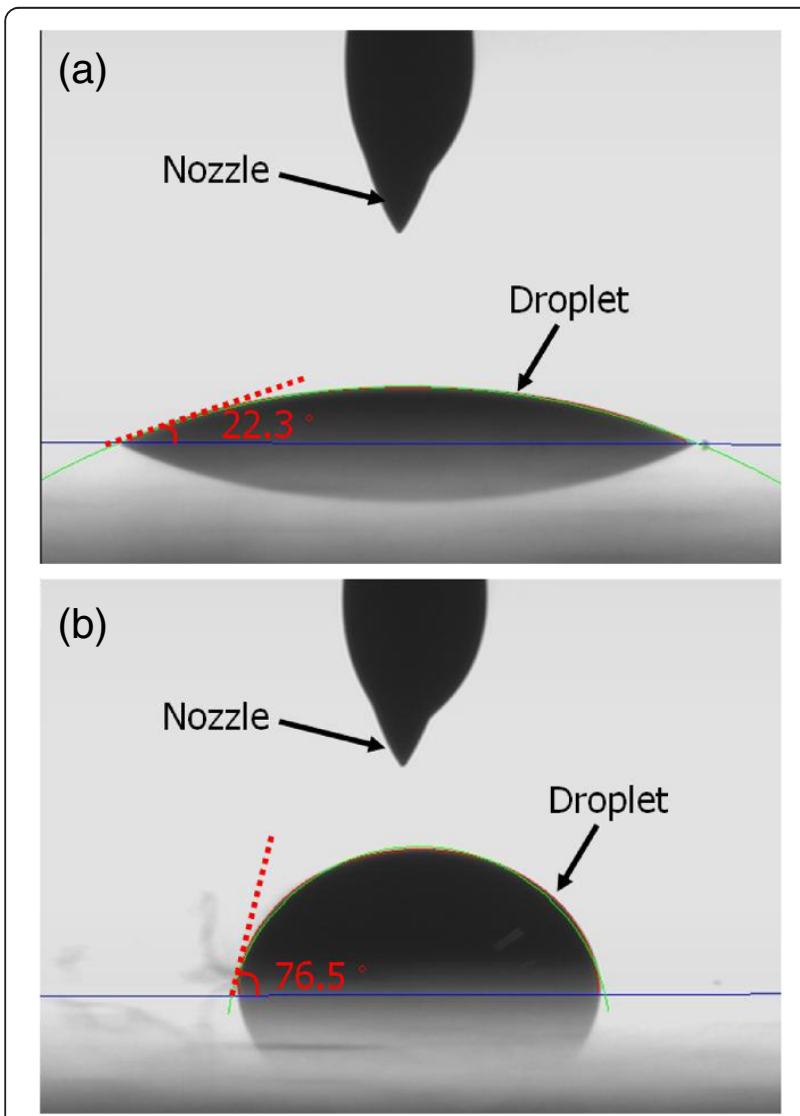

Figure 5 Droplet generation and contact angle measurement according to FC solution treatment on the surface of the substrate. (a) The jetted droplet on the non-FC coated Au surface (b) The jetted droplet on the FC coated surface. wettable printing areas surrounded by a FC film-based inkspreading barrier. To investigate the wettability of Ag ink on the surface, we measured the contact angles of the $\mathrm{Ag}$ inks onto each FC film and Au surface, because the printing area in the proposed fabrication process is affected by the FC film patterning process. As shown in Figure 5, the measured contact angle of the Ag ink surface is quite low, less than $10^{\circ}$ on the Au surface. On the other hand, the $\mathrm{Ag}$
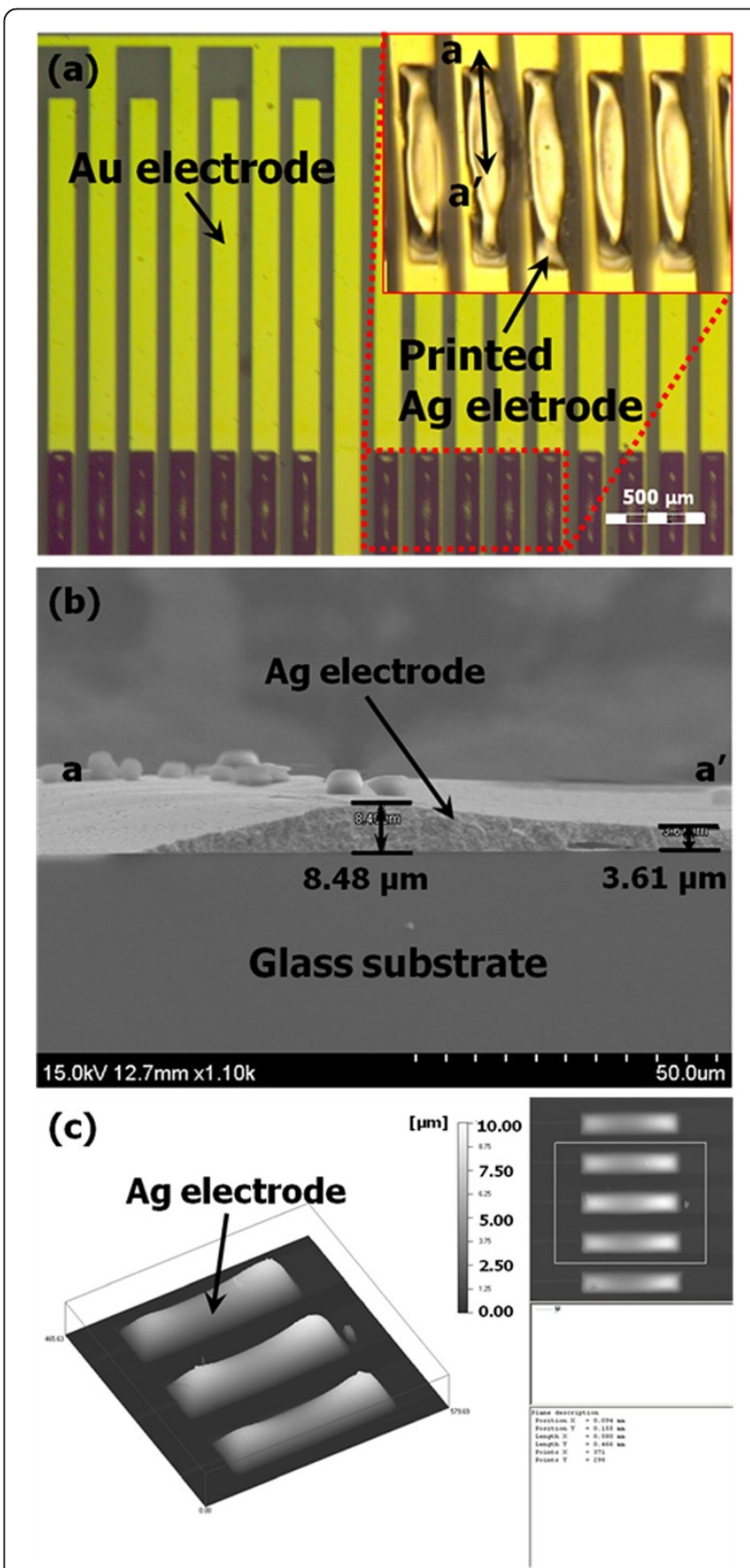

Figure 6 Fabrication results of the 3D Ag electrodes by inkjet printing and FC film patterning. (a) The Au and Ag electrodes fabricated on the substrate, (b) The SEM image of an Ag electrode fabricated on the substrate, (c) The 3D scan images of printed Ag electrodes. 
ink droplet on the FC film surface doesn't spread well and the contact angle is $85.4^{\circ}$, which is much higher than that on the Au surface. These results verified the fact that the hydrophobic FC film can be used as a barrier to control the spreading behavior of the Ag ink on the Au surface.

The inset of Figure 6(a) shows Ag electrodes printed on the $\mathrm{Au}$ electrodes inside a rectangular printing area surrounded by FC film. Ag ink spread and is trapped successfully only on the Au surface without FC film, and FC film acts as a barrier to prevent the ink from over-spreading after the ink-jetting process. However, the thickness of the Ag electrode is not uniform and the edge shape is tilted, as shown in Figure 6(b). The overall morphology is similar to a dented rectangular with round corners (Figure 6(c)). This morphological characteristic would result from the so-called "coffee ring effect" where a ring-like pattern is formed by the accumulation of particles at the edges through capillary flow induced by the differential evaporation rates across the drop. Ag particles inside the printed ink droplet move toward the edges from the center and the particle accumulation at the edge boundaries could result in a dented rectangle with round corners [1]. For further research, the fabrication process needs to be improved to provide rectangular shape of the printed structure by controlling the printing conditions like substrate temperature, surface wettability, and ink vapor pressure $[12,13]$.

Figure 7 shows the DEP characteristic of the $4 \mu \mathrm{m}$ polystyrene beads. The operational voltage was AC $20 \mathrm{~V}_{\mathrm{pp}}$ and the applied frequency was $500 \mathrm{kHz}$. Under these conditions, micro beads represent a negative DEP characteristic on the $\mathrm{Au}$ and $\mathrm{Ag}$ electrodes, because these locations on the electrodes have lower electric field intensity than other locations as shown in the simulation results of Figure 4. In particular, more beads gather on the Ag 3D electrodes since the 3D structure has a broad range of the DEP force as shown in Figure 7(c) and (d). The number of beads trapped on the Ag electrode is 79 and 25 on the Au electrode at the regions (dotted line) indicated by the arrow. The collected beads per unit area are approximately 3 times larger on the $\mathrm{Ag}$ electrode than on the Au electrode. The reason for this is that the electric field distribution is extensive around the 3D electrode, and thus a large amount of the beads can be spatially exposed to the electric field. In the case of the planar electrodes, an electric field distribution is concentrated on the surface of the micro DEP chip, especially at the edges among electrodes, so the electric field influence is much weaker in other areas. Thus, we confirmed that the experimental results have similar trends compared to a prior prediction in the FEM simulation.

\section{Conclusion}

In this work, we successfully demonstrated the new fabrication method for fabricating 3D electrodes by a metal inkjet printing and selective FC film patterning. FC film patterning was very effective at making inkphobic boundaries to prevent droplets from spreading

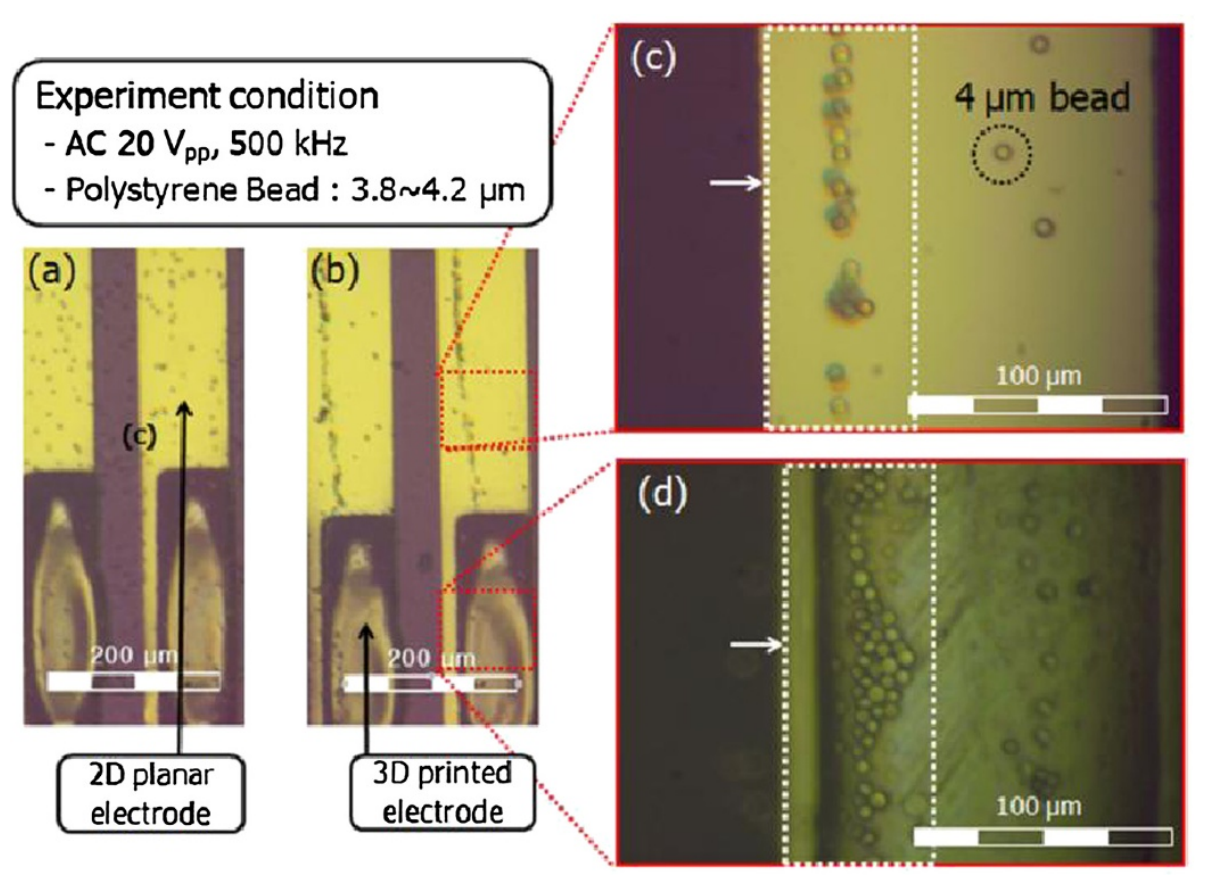

Figure 7 The results of the DEP experiment. (a) Initial state, (b) Applying AC $20 \mathrm{~V}_{\mathrm{pp}}, 500 \mathrm{kHz}$, DEP characteristic of the microbeads (c) on the $2 \mathrm{D}$ electrode, (d) on the 3D printed electrode. 
after the Ag inkjet printing. Ag ink successfully spread and was trapped only on the Au surface without FC film, while the FC films acted as a barrier to prevent the over-spreading after the ink-jetting process. The overall morphology resulted in a dented rectangle with rounded corners due to the coffee ring effect and the thickness of the Ag electrode is non-uniform. However, we could control the thickness of an electrode by regulating the overlap-rate of the inkjet jetting and the number of metal inkjet droplets. The DEP performance of the inkjet-printed Ag electrodes was examined using the $4 \mu \mathrm{m}$ polystyrene beads and AC drive voltage control. We could confirm that more beads were trapped on the 3D electrodes than on the planar electrodes because a large amount of the beads could be spatially exposed to the high electric field in a 3D electrode system. For future research, we need to improve the morphology of the printed structure by controlling printing conditions such as substrate temperature, surface wettability, and ink vapor pressure.

\section{Competing interests}

The authors declare that they have no competing interests.

\section{Authors' contributions}

SHL carried out the experiments, analyzed the experimental results and drafted the manuscript. G-YY performed the metal inkjet printing fabrication. YK, S-HL and Y-KK carried out experimental measurements. All authors read and approved the final manuscript.

\section{Acknowledgements}

This work was supported by the Korea Institute of Industrial Technology.

Received: 23 September 2013 Accepted: 5 December 2013

Published: 18 December 2013

\section{References}

1. Pethig R (2010) Review article-dielectrophoresis: status of the theory, technology, and applications. Biomicrofluidics 4, doi:10.1063/1.3456626

2. Shin KY, Lee SH, Oh JH (2011) Solvent and substrate effects on inkjet-printed dots and lines of silver nanoparticle colloids. J Micromech Microeng 21, doi:10.1088/0960-1317/21/4/045012

3. Burns SE, Cain P, Mills J, Wang JZ, Sirringhaus H (2003) Inkjet printing of polymer thin-film transistor circuits. MRS Bull 28:829-834

4. de Gans BJ, Duineveld PC, Schubert US (2004) Inkjet printing of polymers: state of the art and future developments. Adv Mater 16:203-213

5. Bietsch A, Zhang JY, Hegner M, Lang HP, Gerber C (2004) Rapid functionalization of cantilever array sensors by inkjet printing. Nanotechnology 15:873-880

6. Lee HH, Chou KS, Huang KC (2005) Inkjet printing of nanosized silver colloids. Nanotechnology 16:2436-2441

7. Son Y, Yeo J, Moon H, Lim TW, Hong S, Nam KH, Yoo S, Grigoropoulos CP, Yang DY, Ko SH (2011) Nanoscale electronics: digital fabrication by direct femtosecond laser processing of metal nanoparticles. Adv Mater 23:3176

8. Kim MS, Kim JH, Lee YS, Lim GG, Lee HB, Park JH, Kim YK (2009) Experimental and theoretical analysis of DEP-based particle deflection for the separation of protein-bound particles. J Micromech Microeng 19, doi:10.1088/0960-1317/19/1/015029

9. Iliescu C, Tresset G, Xu GL (2009) Dielectrophoretic field-flow method for separating particle populations in a chip with asymmetric electrodes. Biomicrofluidics 3, doi:10.1063/1.3251125

10. He HQ, Chang DC, Lee YK (2007) Using a micro electroporation chip to determine the optimal physical parameters in the uptake of biomolecules in HeLa cells. Bioelectrochemistry 70:363-368
11. Cheng IF, Froude VE, Zhu YX, Chang HC, Chang HC (2009) A continuous high-throughput bioparticle sorter based on 3D traveling-wave dielectrophoresis. Lab Chip 9:3193-3201

12. Soltman D, Subramanian V (2008) Inkjet-printed line morphologies and temperature control of the coffee ring effect. Langmuir 24:2224-2231

13. de Gans BJ, Schubert US (2004) Inkjet printing of well-defined polymer dots and arrays. Langmuir 20:7789-7793

doi:10.1186/2213-9621-1-5

Cite this article as: Lee et al: Fabrication of a 3 dimensional

dielectrophoresis electrode by a metal inkjet printing method. Micro and Nano Systems Letters 2013 1:5.

\section{Submit your manuscript to a SpringerOpen ${ }^{\circ}$ journal and benefit from:}

- Convenient online submission

$\checkmark$ Rigorous peer review

- Immediate publication on acceptance

- Open access: articles freely available online

- High visibility within the field

- Retaining the copyright to your article

Submit your next manuscript at $\boldsymbol{~ s p r i n g e r o p e n . c o m ~}$ 